Pacific Journal of Mathematics

THE CLOSED PRIME SUBGROUPS OF CERTAIN ORDERED 


\title{
THE CLOSED PRIME SUBGROUPS OF CERTAIN ORDERED PERMUTATION GROUPS
}

\author{
Stephen H. MCCleary
}

\begin{abstract}
The group $G=A(\Omega)$ of all order-preserving permutations of a chain $\Omega$ becomes a lattice-ordered group when ordered pointwise, i.e., $f \leqq g$ if and only if $\beta f \leqq \beta g$ for all $\beta \in \Omega$. Lloyd showed that for each $\omega \in \Omega$, the stabilizer subgroup $G_{\omega}=$ $\{g \in G \mid \omega g=\omega\}$ is a closed prime subgroup of $G$. Our main result (Theorem 11) states that besides $G$ itself, these subgroups, together with the stabilizer subgroups of Dedekind cuts of $\Omega$, comprise all of the closed prime subgroups of $G$.
\end{abstract}

Actually, $G$ need not be all of $A(\Omega)$. In $\S 2$, we use Lloyd's result to show that for depressible or complete subgroups $G$ of $A(\Omega)$, all stabilizer subgroups are closed. We find in $\S 3$ that every closed convex $l$-subgroup $C$ of $G$ is of the form $C=\{g \in G \mid \bar{\Delta} g=\bar{\Delta}\}$ for some collection $\bar{\Delta}$ of points and cuts of $\Omega$. In $\S 4$ we prove the main theorem (stated above) for depressible groups. Finally, this theorem is applied to the question of the extent to which the $l$-group $G$ determines the chain $\Omega$, which was first considered by Holland [4]; and to the determination of the $l$-automorphisms of $G$, considered by Lloyd [5].

2. Stabilizer subgroups. Let $\Omega$ be a chain. A permutation $g$ of $\Omega$ is said to preserve order if $\alpha \leqq \beta$ implies $\alpha g \leqq \beta g$ for all $\alpha, \beta \in \Omega$. The group $A(\Omega)$ of all order-preserving permutations (o-permutations) of $\Omega$, ordered pointwise, is a lattice-ordered group (l-group). $A(\Omega)$ is not assumed to be transitive. For elementary information about $l$ groups, see [1].

$\bar{\Omega}$ will denote the completion of $\Omega$ by Dedekind cuts (without end points unless these end points belong to $\Omega$ ). We shall consider $\Omega$ to be a subchain of $\bar{\Omega}$. Each $g \in A(\Omega)$ can be extended to $\bar{\Omega}$ by defining $\bar{\omega} g(\bar{\omega} \in \bar{\Omega})$ to be $\sup \{\beta g \mid \beta \in \Omega, \beta \leqq \bar{\omega}\}$. Thus $A(\Omega)$ can be considered to be an $l$-subgroup of $A(\bar{\Omega})$, i.e., a subgroup which is also a sublattice.

Let $G$ be an $l$-subgroup of $A(\Omega)$, and thus also of $A(\bar{\Omega}) . \quad G$, or more properly the pair $(G, \Omega)$, is called an ordered permutation group. Holland [3, Th. 2] showed that every abstract $l$-group $L$ is $l$-isomorphic to such a $G$. A subgroup $H$ of $G$ is convex if $h_{1} \leqq g \leqq h_{2}$, with $h_{1}, h_{2} \in H$ and $g \in G$, implies $g \in H$. $H$ is a prime subgroup of $G$ if $H$ is a convex $l$-subgroup of $G$ and if $g_{1} \wedge g_{2}=1$, with $g_{1}, g_{2} \in G$, implies $g_{1} \in H$ or $g_{2} \in H$. For $\bar{\omega} \in \bar{\Omega}$, we define the stabilizer subgroup $G_{\bar{\omega}}$ to be

$$
\{g \in G \mid \bar{\omega} g=\bar{\omega}\},
$$


a prime subgroup of $G$. Thus we have a stabilizer subgroup for each Dedekind cut of $\Omega$ (not just for points of $\Omega$ ).

We wish to consider two classes of $l$-subgroups $G$ of $A(\Omega)$ for which the stabilizer subgroups $G_{\bar{\omega}}$ are closed, i.e., if $s=\sup \left\{s_{i} \mid i \in I\right\}$, with $s \in G$ and $s_{i} \in G_{\bar{\omega}}$ for each $i$ in an index set $I$, then $s \in G_{\bar{\omega}}$.

For $g \in A(\Omega)$ and $\gamma \in \Omega$ such that $\gamma g \neq \gamma$, the interval of support $I(g, \gamma)$ of $g$ which contains $\gamma$ is $\left\{\beta \in \Omega \mid \gamma g^{m} \leqq \beta \leqq \gamma g^{n}\right.$ for some integers $m$ and $n\}$. An $l$-subgroup $G$ of $A(\Omega)$ will be called depressible [7] if it shares with $A(\Omega)$ the following property: If $\Delta$ is an interval of support of $g \in G$, so that $\Delta g=\Delta$, then there exists $h \in G$ such that $\beta h=\beta g$ if $\beta \in \Delta$, but $\beta h=\beta$ if $\beta \notin \Delta$. Intuitively, $h$ is obtained by depressing $g$ outside $\Delta$. As noted in [7], convex $l$-subgroups of $A(\Omega)$ are depressible.

$G$ is a complete l-subgroup of $A(\Omega)$ if whenever $g \in G$ is the sup in $G$ of a collection $\left\{g_{i} \mid i \in I\right\}$ of elements of $G$, then $g$ is also the sup in $A(\Omega)$ of $\left\{g_{i} \mid i \in I\right\}$. By [2, Th. 3.10], an abstract. l-group $L$ is $l$ isomorphic to a complete $l$-subgroup $G$ of some $A(\Omega)$ if and only if $L$ is completely distributive, i.e., if $\bigwedge_{i \in I} \mathbf{V}_{k \in K} g_{i k}=\mathbf{V}_{f \in K^{I}} \Lambda_{i \in I} g_{i f(i)}$ for any collection $\left\{g_{i k} \mid i \in I, k \in K\right\}$ of elements of $G$ for which the indicated sups and infs exist.

THEOREM 1. Suppose $G$ is a depressible or complete l-subgroup of $A(\Omega)$. Then for each $\bar{\omega} \in \bar{\Omega}, G_{\bar{\omega}}$ is a closed prime subgroup of $G$.

Proof. Lloyd [6, Th. 2] proved that if $G=A(\Omega)$, then the stabilizer subgroups $G_{\omega}$ of points $\omega \in \Omega$ are closed. As noted in [7], Lloyd's proof also works when $G$ is a depressible $l$-subgroup of $A(\Omega)$. If $(G, \Omega)$ is depressible, the extension $(G, \bar{\Omega})$ is also depressible. But $G_{\bar{\omega}}$ is the stabilizer subgroup of a point for the group $(G, \bar{\Omega})$, and hence must be closed. On the other hand, suppose that $G$ is complete. If $s=$ $\sup \left\{s_{i} \mid i \in I\right\}$, with $s \in G$ and each $s_{i} \in G_{-}$, then since $G$ is complete, $s$ is also the sup in $A(\Omega)$ of $\left\{s_{i} \mid i \in I\right\}$. Since the stabilizer subgroups are closed for $A(\Omega), \bar{\omega} s=\bar{\omega}$, and then $s \in G_{\bar{\omega}}$. Hence again $G_{\bar{\omega}}$ is closed. (Incidentally, by [7, Th. 7], if $G$ is transitive on $\Omega$, then $G$ is complete if and only if the stabilizer subgroups $G_{\omega}$ are closed.)

A word of warning: $A(\Omega)$, extended to $\bar{\Omega}$, need not be all of $A(\bar{\Omega})$. Thus the above proof, even for $G=A(\Omega)$, does not work without the introduction of the concept of depressible groups.

3. The closed convex $l$-subgroups of $G$. In this section we determine the closed convex $l$-subgroups of $G$, where $G$ is an $l$-subgroup of $A(\Omega)$ which is either depressible or complete. If $C$ is a subgroup of $G$, we define $F x C$ to be $\{\bar{\tau} \in \bar{\Omega} \mid \bar{\tau} C=\bar{\tau}\}$, the collection of points in $\bar{\Omega}$ fixed by $C$. By the convexification $\operatorname{Conv}(\bar{\Delta})$ of a subset $\bar{\Delta}$ of $\bar{\Omega}$, 
we shall mean $\{\bar{\beta} \in \bar{\Omega} \mid \bar{\gamma} \leqq \bar{\beta} \leqq \bar{\delta}$ for some $\bar{\gamma}, \bar{\delta} \in \bar{\Delta}\}$. If $C$ is a subgroup of $G$ and $\bar{\omega} \in \bar{\Omega} \backslash F x C$, we define the orbital of $C$ containing $\bar{\omega}$ to be $\operatorname{Conv}(\bar{\omega} C)$.

Lemma 2. If an orbital $\Gamma$ of $C$ is bounded above, then $\sup \Gamma \in F x C$; and dually.

Proof. $\quad \Gamma C=\Gamma$, so $(\sup \Gamma) C=\sup \Gamma$.

If $L$ is any $l$-group and $C$ is a convex $l$-subgroup of $L$, then the closure $C^{*}$ of $C$ means the smallest closed convex $l$-subgroup of $L$ which contains $C$.

Theorem 3. Suppose that $G$ is a depressible or complete l-subgroup of $A(\Omega)$ and that $C$ is a convex l-subgroup of $G$. Then $C^{*}=$ $\{g \in G \mid \bar{\omega} g=\bar{\omega}$ for all $\bar{\omega} \in F x C\}$.

Proof. Let $D=\{g \in G \mid \bar{\omega} g=\bar{\omega}$ for all $\bar{\omega} \in F x C\}$. Then $D=$ $\bigcap\left\{G_{\bar{\omega}} \mid \bar{\omega} \in F x C\right\}$, so $D$ is a closed convex $l$-subgroup of $G$. Certainly $C^{*} \subseteq D$.

Now pick any $1 \leqq g \in D$ and any $\beta \in \Omega . \quad \operatorname{Conv}\left(\beta C^{*}\right)=\operatorname{Conv}(\beta D)$, for otherwise the lemma would imply that either $\sup \left(\beta C^{*}\right)$ or $\inf \left(\beta C^{*}\right)$ would be an element of $F x C^{*} \backslash F x D$. Hence there exists $f \in C^{*}$ such that $\beta g \leqq \beta f$. Then $1 \leqq(g \wedge f) \vee 1 \leqq f \vee 1 \in C^{*}$, so $(g \wedge f) \vee 1 \in C^{*}$; and $\beta((g \wedge f) \vee 1)=\beta g$. Thus for each $\beta \in \Omega$, there exists an $s_{\beta} \in C^{*}$ such that $\beta s_{\beta}=\beta g$ and $s_{\beta} \leqq g$. Hence $g=\sup \left\{s_{\beta} \mid \beta \in \Omega\right\}$. Since $C^{*}$ is closed, $g \in C^{*}$. Since $g$ was an arbitrary element of $D$, we have shown that $C^{*}=D$.

CoRollary 4. Suppose that $G$ is a depressible or complete l-subgroup of $A(\Omega)$. Then the closed convex l-subgroups of $G$ can be characterized as those subsets $C$ of $G$ for which there exist $\bar{\Delta}_{C} \subseteq \bar{\Omega}$ such that $C=\left\{g \in G \mid \bar{\omega} g=\bar{\omega}\right.$ for all $\left.\bar{\omega} \in \bar{\Delta}_{C}\right\}$. Moreover, every closed convex l-subgroup of $G$ is the intersection of a collection of closed prime subgroups $G_{\bar{\omega}}$ of $G$. Hence the maximal closed convex l-subgroups of $G$ (if any) must be prime.

COROLlaRY 5. Let L be a completely distributive abstract l-group. Then every closed convex l-subgroup of $L$ is the intersection of $a$ collection of closed prime subgroups of $L$; and the maximal closed convex l-subgroup of $L$ (if any) are prime.

Proof. By [2, Th. 3.10], $L$ is $l$-isomorphic to a complete subgroup 
$G$ of some $A(\Omega)$.

4. The closed prime subgroups of $G$. We now develop the tools needed to determine (for the depressible case) that of the closed convex $l$-subgroups of $G$, those which are prime are (besides $G$ itself) precisely the stabilizer subgroups. First we establish some preliminary results which apply to any $l$-subgroup $G$ of $A(\Omega)$. Let us define two binary relations on $\bar{\Omega}: \bar{\omega} F \bar{\tau}(\operatorname{read} \bar{\omega}$ fixes $\bar{\tau})$ if $\bar{\tau} \in F x G_{\bar{\omega}}$, and $\bar{\omega} M \bar{\tau}$ $($ read $\bar{\omega}$ moves $\bar{\tau})$ if $\bar{\tau} \notin F x G_{\bar{\omega}}$.

As a strong hint that the only closed prime subgroups of $G$ are the stabilizer subgroups $G_{\bar{\omega}}$, we have

Proposition 6. If $\bar{\omega} M \bar{\tau}$ and $\bar{\tau} M \bar{\omega}$, then $G_{\bar{i}, \overline{\bar{\tau}}}=G_{\bar{\omega}} \cap G_{\bar{\Sigma}}$ is not prime.

Proof. Since $\bar{\omega} M \bar{\tau}$, we may pick $1<g \in G_{\bar{\omega}}$ such that $\bar{\tau} g>\bar{\tau}$; and since $\bar{\tau} M \bar{\omega}$, we may pick $1<h \in G_{\bar{\tau}}$ such that $\bar{\omega} h>\bar{\omega}$. Then $g \wedge h \in G_{\bar{\omega}, \bar{\tau}}$ while neither $g$ nor $h$ lies in $G_{\dot{\omega}, \overline{\bar{\nu}}}$, so $G_{\bar{\omega}, \overline{\bar{\tau}}}$ is not prime.

Proposition 7. $\bar{\omega} F \bar{\tau}$ if and only if $G_{\bar{\omega}} \leqq G_{\overline{\bar{*}}}$. Hence $G_{\bar{\omega}}=G_{\bar{\gamma}}$ if and only if $\bar{\omega} F \bar{\tau}$ and $\bar{\tau} F \bar{\omega}$.

Lemma 8. Suppose $\Gamma$ is an orbital of $G_{\bar{\eta}}, \bar{\tau} \in \bar{\Omega}$. Then for $g \in G$, $\Gamma g$ is an orbital of $G_{\bar{q} g}$.

Proof. $\quad \Gamma=\operatorname{Conv}\left(\bar{\omega} G_{\bar{\tau}}\right)$ for some $\bar{\omega} \in \bar{\Omega}$, so $\Gamma g=\operatorname{Conv}(\bar{\omega} G=g)=$ $\operatorname{Conv}\left((\bar{\omega} g)\left(g^{-1} G_{\bar{\tau}} g\right)\right)=\operatorname{Conv}\left((\bar{\omega} g) G_{\bar{\tau} g}\right)$.

Lemma 9. Suppose $\Gamma$ is an orbital of $G_{\bar{\eta}}, \bar{\tau} \in \bar{\Omega}$. If $\bar{\tau} g=\bar{\tau} h$, with $g, h \in G$, then $\Gamma g=\Gamma h$.

Proof. If $\bar{\tau} g=\bar{\tau} h$, then $g h^{-1} \in G_{\bar{\tau}}$, so $\Gamma g h^{-1}=\Gamma$ (since $\Gamma$ is an orbital of $G_{\bar{\tau}}$ ), and thus $\Gamma g=\Gamma h$.

By an o-block of $G$, or more properly of $(G, \bar{\Omega})$, we mean a nonempty convex subset $\bar{\Delta}$ of $\bar{\Omega}$ such that for any $g \in G$, either $\bar{\Delta} g=\bar{\Delta}$ or $\bar{\Delta} g \cap \bar{J}=\square$. ( $\square$ denotes the empty set.) An o-block is trivial if it contains only one point.

Lemma 10. If $\bar{\omega} F \bar{\tau}$, but $\bar{\tau} M \bar{\omega}$, then the orbital $\Gamma=\operatorname{Conv}\left(\bar{\omega} G_{\bar{\tau}}\right)$ of $G_{\bar{\tau}}$ is a nontrivial o-block of $G$. For $g \in G, \Gamma g=\Gamma$ if and only if $g \in G_{\bar{\tau}}$.

Proof. If $g \in G_{\bar{\tau}}$, then $\Gamma g=\Gamma 1=\Gamma$ by Lemma 9. We now show that if $\bar{\tau}<\bar{\tau} g$, then $\Gamma g \cap \Gamma=\square$. If not, we use Lemma 8 to pick 
$h \in G_{\bar{\tau} g}$ such that $(\bar{\omega} g) h \in \Gamma g \cap \Gamma$, and then we pick $k \in G_{\bar{\tau}}$ such that $(\bar{\omega} g h) k \leqq \bar{\omega}$. Then $\bar{\omega}(g h k \vee 1)=\bar{\omega}$, but $\bar{\tau}<\bar{\tau} g=\bar{\tau} g h$, so $\bar{\tau}=\bar{\tau} k<$ $\bar{\tau} g h k \leqq \bar{\tau}(g h k \vee 1)$. This contradicts the assumption that $\bar{\omega} F \bar{\tau}$. Similarly, if $\bar{\tau}>\bar{\tau} g, \Gamma g \cap \Gamma=\square$.

MaIn Theorem 11. Suppose $G$ is a depressible l-subgroup of $A(\Omega)$. Then the closed prime subgroups of $G$ (besides $G$ itself) are precisely the stabilizer subgroups $G_{\bar{\omega}}, \bar{\omega} \in \bar{\Omega}$.

Proof. By Theorem 1, the $G_{\bar{\omega}}$ 's are closed prime subgroups of $G$. Now let $C$ be any closed prime subgroup of $G$. By Corollary $4, C=$ $\{g \in G \mid \bar{\omega} g=\bar{\omega}$ for all $\bar{\omega} \in F x C\}$. If $F x C=\square, C=G$. Now suppose $F x C \neq \square$. For each $\bar{\omega} \in F x C, C \leqq G_{\bar{\omega}}$. In any l-group, the collection of convex $l$-subgroups containing a given prime subgroup is a chain under inclusion [3, Lemma 3], so $\mathscr{S}=\left\{G_{\bar{\omega}} \mid \bar{\omega} \in F x C\right\}$ is a chain. Moreover $\cap \mathscr{S}=C$.

If for $G_{\bar{\omega}}, G_{\bar{\tau}} \in \mathscr{S}$, we have $G_{\bar{\omega}} \subset G_{\bar{\tau}}$ (i.e., if $\bar{\omega} F \bar{\tau}$, but $\bar{\tau} M \bar{\omega}$ ), then by Lemma $10, \bar{\Delta}(\bar{\omega}, \bar{\tau})=\operatorname{Conv}\left(\bar{\omega} G_{\bar{\tau}}\right)$ is a nontrivial o-block of $G$. We show next that $\bar{\Delta}(\bar{\omega}, \bar{\tau})$ is independent of $\bar{\omega}$. For suppose that also $G_{\bar{\sigma}} \in \mathscr{S}$, and $G_{\bar{\sigma}} \subseteq G_{\bar{\omega}}$. Then $\bar{\sigma} \in \bar{\Delta}(\bar{\omega}, \bar{\tau})$, for otherwise we could pick $h \in G_{\bar{\tau}}$ such that $\bar{\omega} h \neq \bar{\omega}$ and depress $h$ outside $\bar{\Delta}(\bar{\omega}, \bar{\tau})$ to obtain an element of $G_{\bar{\sigma}}$ which moves $\bar{\omega}$, contradicting the fact that $\bar{\sigma} F \bar{\omega}$. Since $\bar{\sigma} \in \bar{\Delta}(\bar{\omega}, \bar{\tau}), \bar{\Delta}(\bar{\sigma}, \bar{\tau})=\operatorname{Conv}\left(\bar{\sigma} G_{\bar{\tau}}\right)=\operatorname{Conv}\left(\bar{\omega} G_{\bar{\tau}}\right)=\bar{\Delta}(\bar{\omega}, \bar{\tau})$. Hence we may write $\bar{\Delta}(\bar{\omega}, \bar{\tau})$ simply as $\bar{\Delta}(\bar{\tau})$; and $\left\{\bar{J}(\bar{\tau}) \mid G_{\bar{\gamma}} \in \mathscr{S}\right\}$ forms a tower under inclusion. Let $\bar{\delta}(\bar{\tau})=\sup \bar{\Delta}(\bar{\tau})$ and let $\bar{\xi}=\inf \left\{\bar{\delta}(\bar{\tau}) \mid G_{\bar{\tau}} \in \mathscr{S}\right\}$. Since $C$ fixes each $\bar{\tau}$ such that $G_{\bar{\tau}} \in \mathscr{S}$, it fixes each $\bar{\Delta}(\bar{\tau})$ (by Lemma 10) and thus each $\bar{\delta}(\bar{\tau})$, so it fixes $\bar{\xi}$. Conversely, since $\bar{\sigma} \in \bar{\Delta}(\bar{\tau})$ whenever $G_{\bar{\sigma}} \subset G_{\overline{\bar{z}}}$, we have $\bar{\xi} \in \bar{\Delta}(\bar{\tau})$. Since also $\bar{\Delta}(\bar{\tau})$ is an o-block of $G, G_{\bar{\xi}}$ fixes each $\bar{\Delta}(\bar{\gamma})$. Hence by Lemma $10, G_{\bar{\xi}}$ fixes each $\bar{\tau}$. Therefore $G_{\bar{\xi}}=\{g \in G \mid \bar{\omega} g=\bar{\omega}$ for all $\bar{\omega} \in F x C\}=C$. This concludes the proof.

Theorem 11 fails when completeness is substituted for depressibility, even under the additional assumption that $G$ is transitive. However, examples of this phenomenon are too complicated to be presented here. The author is presently trying to find additional hypotheses under which the modified theorem will hold.

By Zorn's lemma, every closed prime subgroup of $G$ contains a minimal closed prime subgroup of $G$, which is characterized by

COROLLARY 12. $G_{\bar{\omega}}$ is a minimal closed prime subgroup of $G$ if and only if for all $\bar{\tau} \in \bar{\Omega}, \bar{\omega} M \bar{\tau}$ implies $\bar{\tau} M \bar{\omega}$.

We define $\operatorname{Min}(\bar{\Omega})$ to be $\left\{\bar{\omega} \in \bar{\Omega} \mid G_{\bar{\omega}}\right.$ is a minimal closed prime subgroup of $G$ \}.

Theorem 13. Suppose that $G$ is a depressible l-subgroup of $A(\Omega)$ 
and that for each $\alpha \in \Omega, G_{\alpha}$ has no fixed points in $\Omega$ except $\alpha$. Then $\left\{G_{\bar{\omega}} \mid \bar{\omega} \in \operatorname{Min}(\bar{\Omega})\right\}$ are distinct and include all $G_{\alpha}$ 's, $\alpha \in \Omega$. Moreover, $\bar{\omega} \in \operatorname{Min}(\bar{\Omega})$ if and only if $\bar{\tau} M \bar{\omega}$ for every $\bar{\omega} \neq \bar{\tau} \in \bar{\Omega}$.

Proof. Suppose that $G_{\bar{\omega}} \subseteq G_{\alpha}$, with $\alpha \in \Omega$ and $\alpha \neq \bar{\omega} \in \bar{\Omega}$. If $\bar{\omega} \in \Omega$, then $\bar{\omega} F \alpha$ contradicts our hypothesis. Hence $\bar{\omega} \in \bar{\Omega} \backslash \Omega$, so we may pick $\beta \in \Omega$ lying strictly between $\alpha$ and $\bar{\omega}$. Then by hypothesis, we may pick $h \in G_{\beta}$ such that $\alpha h \neq \alpha$. By depressing $h$ outside the interval of support $I(h, \alpha)$, we obtain an element of $G$ which moves $\alpha$, but fixes $\bar{\omega}$; contradicting the assumption that $G_{\bar{\omega}} \subseteq G_{\alpha}$. This shows that $G_{\alpha}$ is minimal, and moreover that $G_{\alpha} \neq G_{\bar{\omega}}$ for $\alpha \neq \bar{\omega} \in \bar{\Omega}$.

Now let $\bar{\omega}<\bar{\tau}$, with $\bar{\omega}, \bar{\tau} \in \operatorname{Min}(\bar{\Omega})$, and suppose that $G_{\bar{\omega}}=G_{\bar{\tau}}$. Pick $\alpha \in \Omega$ such that $\bar{\omega}<\alpha \leqq \bar{\tau}$. Since $G_{\bar{\omega}}$ is minimal, $G_{\alpha} \not \subset G_{\bar{\omega}}$, and by the previous paragraph, $G_{\alpha} \neq G_{\bar{\omega}}$. Hence we may pick $k \in G_{\alpha}$ such that $\bar{\omega} k \neq \bar{\omega}$. By depressing $k$ outside $I(k, \bar{\omega})$, we obtain an element of $G$ which moves $\bar{\omega}$, but fixes $\bar{\tau}$; contradicting the assumption that $G_{\bar{\omega}}=G_{\overline{-}}$. Hence $\left\{G_{\bar{\omega}} \mid \bar{\omega} \in \operatorname{Min}(\bar{\Omega})\right\}$ are distinct. The last part of the theorem now follows from Corollary 12.

5. The relation between the $l$-group $G$ and the chain $\Omega$. Suppose now that the hypotheses of the last theorem are satisfied. We wish to determine how the chain $\operatorname{Min}(\bar{\Omega})$ is reflected in the $l$-group structure of $G$. The theorem gives us a one-to-one correspondence $\bar{\omega} \leftrightarrow G_{\bar{\omega}}$ between $\operatorname{Min}(\bar{\Omega})$ and the collection of minimal closed prime subgroups of $G$. Ideally, we would like to use the $l$-group structure of $G$ to order these minimal closed prime subgroups so as to make this correspondence be an o-isomorphism (preserve order both ways). In general, this program will only partially succeed.

$(G, \Omega)$ and $(H, \Sigma)$ are said to be isomorphic as o-permutation groups if there is an $o$-isomorphism $\theta$ from $\Omega$ onto $\Sigma$ and a map $\psi$ from $G$ onto $H$ such that $(\alpha g) \theta=(\alpha \theta)(g \psi)$ for all $\alpha \in \Omega, g \in G$. (It then follows that $\psi$ is an $l$-isomorphism.) By the characteristic chains of $(G, \Omega)$ we shall mean the orbits $\bar{\omega} G$ of $G$ (no convexification this time), for which $\bar{\omega} \in \operatorname{Min}(\bar{\Omega})$. Of course, if $\bar{\omega} \in \operatorname{Min}(\bar{\Omega})$, then $\bar{\omega} G \leqq \operatorname{Min}(\bar{\Omega})$ since $G_{\bar{\omega} g}=g^{-1} G_{\bar{u}} g$. The characteristic chains of $(G, \Omega)$ partition $\operatorname{Min}(\bar{\Omega})$, and a subcollection of them partitions $\Omega$. In particular, $(\operatorname{Min}(\bar{\Omega})) G=$ $\operatorname{Min}(\bar{\Omega})$, and $G$ is faithful on $\operatorname{Min}(\bar{\Omega})$ since $\Omega \subseteq \operatorname{Min}(\bar{\Omega})$. The closure of a characteristic chain will refer to the order topology of Min $(\bar{\Omega})$; or equivalently, since $\operatorname{Min}(\bar{\Omega})$ is dense in $\bar{\Omega}$, to the order topology of $\bar{\Omega}$. The closure of any one characteristic chain is of course a union of characteristic chains.

THeorem 14. Suppose that $(G, \Omega)$ and $(H, \Sigma)$ both satisfy the hypotheses of Theorem 13, and that $\psi$ is an l-group isomorphism 
from $G$ onto $H$. Then there is an o-isomorphism $\theta$ from $\operatorname{Min}(\bar{\Omega})$ onto $\operatorname{Min}(\bar{\Sigma})$ such that $\theta$ and $\psi$ provide an o-permutation group isomorphism from $(G, \Omega)$ onto $(H, \Sigma)$. (Exception: The order of $\bar{\sigma}$ and $\bar{\tau}$ need not be preserved unless there is some characteristic chain of $(G, \Omega)$ whose closure contains them both.) $\theta$ preserves characteristic chains and closures of characteristic chains. With the above exception, $\Sigma$ is o-isomorphic to the union $\bar{\Gamma}$ of a collection of characteristic chains of $(G, \Omega)$, and $(H, \Sigma)$ is isomorphic as an o-permutation group to $(G, \bar{\Gamma})$.

Proof. We wish to (almost) determine the order of $\operatorname{Min}(\bar{\Omega})$ from the $l$-group structure of $G$. Since $G_{\bar{\omega}}(\bar{\omega} \in \operatorname{Min}(\bar{\Omega}))$ is a prime subgroup of $G$, we can totally order the set $R\left(G_{\bar{\omega}}\right)$ of $\operatorname{cosets} G_{\bar{\omega}} g(g \in G)$ by defining $G_{\bar{\omega}} g \leqq G_{\bar{\omega}} k$ if and only if there exist $s \in G_{\bar{\omega}} g$ and $t \in G_{\bar{\omega}} k$ such that $s \leqq t$ [3, Lemma 4]. It is easily checked that the correspondence $G_{\bar{\omega}} g \leftrightarrow \bar{\omega} g$ is an $o$-isomorphism between $R\left(G_{\bar{\omega}}\right)$ and the characteristic chain $\bar{\omega} G$. Moreover, $\left(G_{\bar{\omega}} g\right) k=G_{\bar{\omega}}(g k) \leftrightarrow \bar{\omega}(g k)=(\bar{\omega} g) k, k \in G$.

Now let $\bar{\Delta}$ be a characteristic chain of $(G, \Omega)$ and let $\left(\bar{\Delta}_{1}, \bar{\Delta}_{2}\right)$ be a Dedekind cut of $\bar{\Delta}$ such that $\bar{\Delta}_{1}$ has no greatest element and $\bar{\Delta}_{2}$ no least element. Let $\bar{\delta}_{1}$ be the sup in $\bar{\Omega}$ of $\bar{\Delta}_{1}$ and $\bar{\delta}_{2}$ the inf in $\bar{\Omega}$ of $\bar{\Delta}_{2}$. Let $G^{\prime}=\left\{g \in G \mid \bar{\Delta}_{1} g=\bar{\Delta}_{1}\right.$ and $\left.\bar{\Delta}_{2} g=\bar{\Delta}_{2}\right\}$. Then $G_{\bar{\delta}_{1}}=G^{\prime}=G_{\bar{\delta}_{2}}$. Hence $G^{\prime}$ is a minimal closed prime subgroup of $G$ if and only if there exists $\bar{\tau} \in \operatorname{Min}(\bar{\Omega})$ lying in the closure of $\bar{\Delta}$ and satisfying $\bar{\Delta}_{1}<\bar{\tau}<\bar{\Delta}_{2}$. (If there exists such a $\bar{\tau}$, then $\bar{\tau}=\bar{\delta}_{1}$ or $\bar{\delta}_{2}$, say $\bar{\delta}_{1}$; so that $G^{\prime}=G_{\bar{\delta}_{1}}=G_{\bar{\tau}}$ is minimal.) Moreover, if $G^{\prime}$ is minimal, then since $G_{\bar{\delta}_{1}}=G_{\bar{\delta}_{2}}$, Theorem 13 guarantees that $\bar{\delta}_{1}=\bar{\delta}_{2}$ and thus that $\bar{\tau}$ is unique. Thus the closure of $\bar{\Delta}$ in $\operatorname{Min}(\bar{\Omega})$ is determined by the l-group structure of $G$. (Of course, neither the sup nor the inf in $\bar{\Omega}$ of a nontrivial $\bar{\Delta}$ can lie in $\operatorname{Min}(\bar{\Omega})$.) Similar considerations apply to $(H, \Sigma)$. For $\bar{\omega} \in \operatorname{Min}(\bar{\Omega})$, define $\bar{\omega} \theta$ to be the point in $\operatorname{Min}(\bar{\Sigma})$ stabilized by $G_{\bar{\omega}} \psi$. The theorem follows.

The limitations of the theorem are illustrated by the following example. Let $\Sigma_{\alpha}$ be an $\alpha$-set, $\alpha=0,1,2$. (An $\alpha$-set is a chain $\Sigma$ of cardinality $\boldsymbol{\aleph}_{\alpha}$ in which for any two subsets $\Gamma<\Delta$ of cardinality less than $\boldsymbol{\aleph}_{\alpha}$, there exists $\sigma \in \Sigma$ such that $\Gamma<\sigma<\Delta$.) Let $\Pi_{1}$ be the ordinal sum $\Sigma_{0} \oplus \Sigma_{1} \oplus \Sigma_{2}$, and $\Pi_{2}$ the ordinal sum $\Sigma_{0} \oplus \Sigma_{2} \oplus \Sigma_{1}$. Let $\Omega_{i}, i=1,2$, be the lexicographic product $\overleftarrow{\Pi_{i} \times J}$, ordered from the right, where $J$ denotes the integers. Each copy of each $\Sigma_{\alpha}$ is an o-block of each $A\left(\Omega_{i}\right)$, whence it is easily seen that $A\left(\Omega_{1}\right)$ and $A\left(\Omega_{2}\right)$ are isomorphic as $l$-groups, despite the fact that $\operatorname{Min}\left(\bar{\Omega}_{1}\right)$ and $\operatorname{Min}\left(\bar{\Omega}_{2}\right)$ are not $o$-isomorphic.

COROLlaRY 15. If the l-group $G$ has at least one transitive representation $(K, \Pi)$ of the sort described in the theorem, then the exception in the theorem can be removed. 
Proof. Since $K$ is transitive, $\Pi$ is a characteristic chain of $(K, \Pi)$. The closure of $I$ in Min $(\bar{\Pi})$ is the entire chain Min $(\bar{\Pi})$. The theorem yields genuine $o$-isomorphisms from $\operatorname{Min}(\bar{\Pi})$ onto $\operatorname{Min}(\bar{\Omega})$, and also from $\operatorname{Min}(\bar{\Pi})$ onto $\operatorname{Min}(\bar{\Sigma})$.

For the special case in which $G$ and $H$ are themselves transitive and have minimal nontrivial $o$-blocks, this corollary is precisely Holland's main result in [4, Th. 7], applied to the depressible case. (Holland's result does not assume depressibility and is proved without the use of closed subgroups.) Further exploration of the transitive case takes on rather a different flavor and will be pursued in a later paper.

In [3, Th. 2], Holland represents an arbitrary l-group $L$ as an $l$-subgroup $G$ of an appropriate $A(\Omega)$, where the chain $\Omega$ is partitioned into convex subsets $\Omega_{i}$ such that for each $\Omega_{i}, \Omega_{i} G=\Omega_{i}$ and $G$ is transitive on $\Omega_{i}$. If one such representation $(G, \Omega)$ satisfies the hypotheses of Theorem 13 (equivalently, if for each $\Omega_{i}$, the restriction $\left(G \mid \Omega_{i}, \Omega_{i}\right)$ satisfies the hypotheses of Theorem 13), then up to $o$-isomorphism, every such representation satisfying those hypotheses involves the same chains $\operatorname{Min}\left(\bar{\Omega}_{i}\right)$.

Corollary 16. Suppose that $(G, \Omega)$ satisfies the hypotheses of Theorem 13. Then every l-automorphism \& of $G$ is induced by an o-permutation $\theta$ of $\operatorname{Min}(\bar{\Omega})$, i.e., $g \psi=\theta^{-1} g \theta$ for all $g \in G$. Moreover, $\theta$ permutes the collection of characteristic chains of $(G, \Omega)$ and preserves their closures. $\theta$ is subject to the exception of Theorem 14. This exception can be removed under the additional assumption of Corollary 15 , and then the present result states that every l-automorphism of $G$ is induced by an inner automorphism of $A(\operatorname{Min}(\bar{\Omega}))$.

Proof. $(G, \Omega)$ and $(G \psi, \Omega)$ satisfy the hypotheses of Theorem 14 . Let $\theta$ be the $o$-permutation of $\operatorname{Min}(\bar{\Omega})$ provided by that theorem. Then $(\bar{\omega} \theta)(g \psi)=(\bar{\omega} g) \theta$ for all $\bar{\omega} \in \operatorname{Min}(\bar{\Omega}), g \in G$, i.e., $\theta g \psi=g \theta$, and thus $g \psi=\theta^{-1} g \theta$.

Lloyd [5, Th. 1.10] proved Corollary 16 for the case in which $G$ is all of $A(\Omega)$ and is transitive, except that $\theta$ was an $o$-permutation of $\bar{\Omega}$, rather than of the smaller chain Min $(\bar{\Omega})$. Lloyd's proof did not make use of closed subgroups. In many specific cases (e.g., if $\Omega$ is Dedekind complete or is the chain of rational numbers), the last statement in the corollary permits one to deduce (following Lloyd) that every $l$-automorphism of $A(\Omega)$ is inner.

\section{REFERENCES}

1. G. Birkhoff, Lattice theory, $3^{\text {rd }}$ ed., Amer. Math. Soc. Colloq. Publns., Vol. 25, Providence, R.I., 1967.

2. R. D. Byrd and J. T. Lloyd, Closed subgroups and complete distributivity in lattice- 
ordered groups, Math. Zeit. 101 (1967), 123-130.

3. C. Holland, The lattice-ordered group of automorphisms of an ordered set, Michigan Math. J. 10 (1963), 399-408.

4. , Transitive lattice-ordered permutation groups, Math. Zeit 87 (1965), 420433.

5. J. T. Lloyd, Lattice-ordered groups and o-permutations groups, Dissertation, Tulane University, 1964.

6. Complete distributivity in certain infinite permutation groups, Michigan Math. J. 14 (1967), 393-400.

7. S. H. McCleary, Pointwise suprema of order-preserving permutations (to appear in Illinois J. Math.)

Received June 19, 1969.

UNIVERSity of Georgia

Athens, Georgia 



\title{
PACIFIC JOURNAL OF MATHEMATICS
}

\author{
EDITORS
}

\author{
H. ROYDEN \\ Stanford University \\ Stanford, California \\ RICHARD PIERCE \\ University of Washington \\ Seattle, Washington 98105
}

\author{
J. DUGUNDJI \\ Department of Mathematics \\ University of Southern California \\ Los Angeles, California 90007 \\ BASIL GORDON \\ University of California \\ Los Angeles, California 90024
}

\section{ASSOCIATE EDITORS}

E. F. BECKENBACH
B. H. NeumanN

F. WOLF
K. YoshidA

\section{SUPPORTING INSTITUTIONS}

\author{
UNIVERSITY OF BRITISH COLUMBIA \\ CALIFORNIA INSTITUTE OF TECHNOLOGY \\ UNIVERSITY OF CALIFORNIA \\ MONTANA STATE UNIVERSITY \\ UNIVERSITY OF NEVADA \\ NEW MEXICO STATE UNIVERSITY \\ OREGON STATE UNIVERSITY \\ UNIVERSITY OF OREGON \\ OSAKA UNIVERSITY \\ UNIVERSITY OF SOUTHERN CALIFORNIA
}

\author{
STANFORD UNIVERSITY \\ UNIVERSITY OF TOKYO \\ UNIVERSITY OF UTAH \\ WASHINGTON STATE UNIVERSITY \\ UNIVERSITY OF WASHINGTON

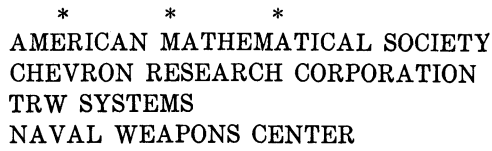

The Supporting Institutions listed above contribute to the cost of publication of this Journal, but they are not owners or publishers and have no responsibility for its content or policies.

Mathematical papers intended for publication in the Pacific Journal of Mathematics should be in typed form or offset-reproduced, double spaced with large margins. Underline Greek letters in red, German in green, and script in blue. The first paragraph or two must be capable of being used separately as a synopsis of the entire paper. It should not contain references to the bibliography. Manuscripts, in duplicate if possible, may be sent to any one of the four editors. Please classify according to the scheme of Math. Rev. 36, 1539-1546. All other communications to the editors should be addressed to the managing editor, Richard Arens, University of California, Los Angeles, California, 90024.

50 reprints are provided free for each article; additional copies may be obtained at cost in multiples of 50 .

The Pacific Journal of Mathematics is published monthly. Effective with Volume 16 the price per volume (3 numbers) is $\$ 8.00$; single issues, $\$ 3.00$. Special price for current issues to individual faculty members of supporting institutions and to individual members of the American Mathematical Society: $\$ 4.00$ per volume; single issues $\$ 1.50$. Back numbers are available.

Subscriptions, orders for back numbers, and changes of address should be sent to Pacific Journal of Mathematics, 103 Highland Boulevard, Berkeley, California, 94708.

PUBLISHED BY PACIFIC JOURNAL OF MATHEMATICS, A NON-PROFIT CORPORATION

Printed at Kokusai Bunken Insatsusha (International Academic Printing Co., Ltd.), 7-17, Fujimi 2-chome, Chiyoda-ku, Tokyo, Japan. 


\section{Pacific Journal of Mathematics}

\section{Vol. 31, No. $3 \quad$ BadMonth, 1969}

George E. Andrews, On a calculus of partition functions .................. 555

Silvio Aurora, A representation theorem for certain connected rings ............ 563

Lawrence Wasson Baggett, A note on groups with finite dual spaces ............. 569

Steven Barry Bank, On majorants for solutions of algebraic differential equations in regions of the complex plane ............................... 573

Klaus R. Bichteler, Locally compact topologies on a group and the corresponding continuous irreducible representations ......................... 583

Mario Borelli, Affine complements of divisors ....................... 595

Carlos Jorge Do Rego Borges, A study of absolute extensor spaces ............. 609

Bruce Langworthy Chalmers, Subspace kernels and minimum problems in Hilbert

spaces with kernel function ...................................... 619

John Dauns, Representation of L-groups and F-rings................. 629

Spencer Ernest Dickson and Kent Ralph Fuller, Algebras for which every

indecomposable right module is invariant in its injective envelope ...........

Robert Fraser and Sam Bernard Nadler, Jr., Sequences of contractive maps and fixed

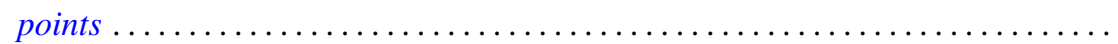

Judith Lee Gersting, A rate of growth criterion for universality of regressive

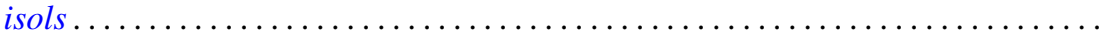

Robert Fred Gordon, Rings in which minimal left ideals are projective ............

Fred Gross, Entire functions of several variables with algebraic derivatives at certain algebraic points

W. Charles (Wilbur) Holland Jr. and Stephen H. McCleary, Wreath products of ordered permutation groups .........................

W. J. Kim, The Schwarzian derivative and multivalence .................. 717

Robert Hamor La Grange, Jr., On $(\mathrm{m}-\mathrm{n})$ products of Boolean algebras ......... 725

Charles D. Masiello, The average of a gauge ........................ 733

Stephen H. McCleary, The closed prime subgroups of certain ordered permutation

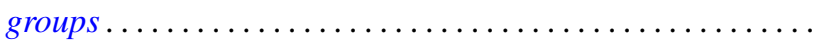

Richard Roy Miller, Gleason parts and Choquet boundary points in convolution measure algebras ...............................

Harold L. Peterson, Jr., On dyadic subspaces ........................ 773

Derek J. S. Robinson, Groups which are minimal with respect to normality being intransitive........................................... 777

Ralph Edwin Showalter, Partial differential equations of Sobolev-Galpern type . . . 787

David Slepian, The content of some extreme simplexes ................... 795

Joseph L. Taylor, Noncommutative convolution measure algebras ............. 809

B. S. Yadav, Contractions of functions and their Fourier series ............... 827

Lindsay Nathan Childs and Frank Rimi DeMeyer, Correction to automorphisms of separable algebras" ....................... 833

Moses Glasner and Richard Emanuel Katz, Correction to: "Function-theoretic degeneracy criteria for Riemannian manifolds".............

Satish Shirali, Correction to: "On the Jordan structure of complex Banach

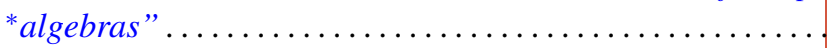

